\title{
An Infrared Quantitative Imaging Technique (IR-QIV) for Remote Sensing of River Flows
}

\author{
Edwin A. Cowen ${ }^{1 *}$, Seth A. Schweitzer ${ }^{1}$ \\ ${ }^{1}$ DeFrees Hydraulics Laboratory, School of Civil \& Environmental Engineering, Cornell University, \\ Ithaca, NY*eac20@cornell.edu
}

Stage and discharge are some of the oldest measurements in environmental fluid mechanics and are vital in forecasting water supply and flood safety. These measurements are traditionally manpower intensive, hence expensive, and dangerous under high flow conditions. Considering climate change and the planet's increasing population there is a critical need for better, more accurate, and frequent, in space and time, data for model and forecast guidance. This need spans monitoring small-scale turbulent processes to calibrating and nudging continental scale river dynamics models. Driven by applications from river gaging networks to fish behavior modeling to flood and erosion forecasting, and more generally, the near-shore environment of lakes, estuaries and the coasts, remote sensing with quantitative imaging tools is a rapidly expanding field. Such tools can be deployed from fixed platforms, drones, planes and satellites with valuable information contained within the visible to infrared spectral bands.

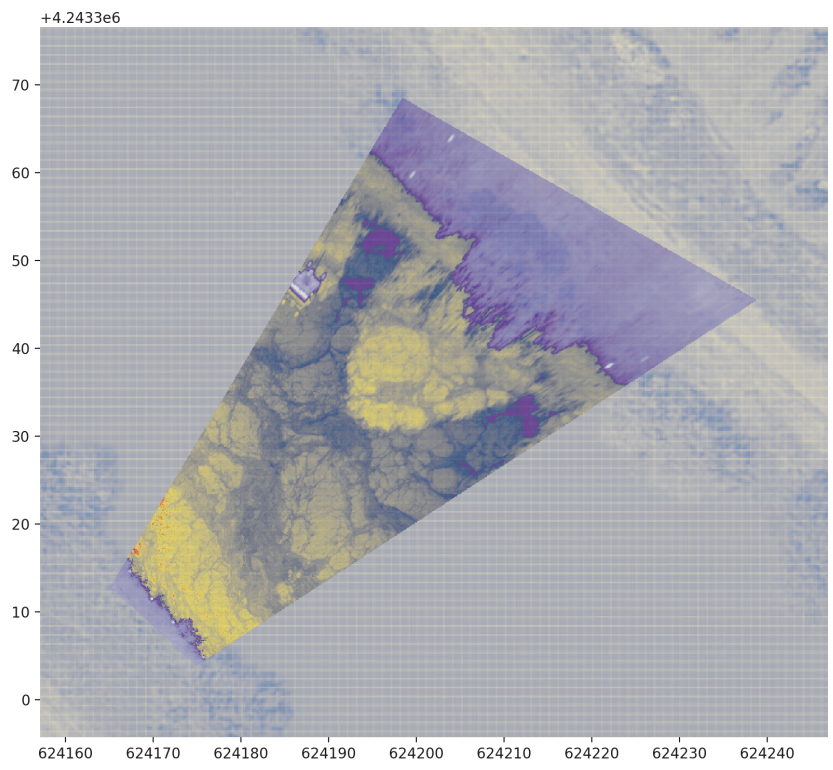

Figure 1: Infrared image of the water surface of a river (Sutter Slough, California, USA). The infrared image is georeferenced and overlaid on an aerial image. Detailed thermal patterns can be seen at the water surface. Yellow and blue hues indicate thermal patterns at the water surface, purple and silver hues are from foliage on the river banks. The width of the river is approximately $50 \mathrm{~m}$. Distances are in meters on the UTM grid.
In this presentation we focus on using a remotely mounted infrared (IR) camera to monitor the mean and instantaneous surface velocity field of rivers and on how bathymetry, flow rate, and metrics of turbulence, can be inferred from the collected IR images. We provide details of our developed infrared quantitative image velocimetry (IR-QIV) method, which capable of measuring instantaneous velocity at high spatial and temporal resolution, over spatial domains with side length scales of order $10 \mathrm{~m}$ to $100 \mathrm{~m}$ (i.e., areas of $10^{2} \mathrm{~m}^{2}$ to $10^{4} \mathrm{~m}^{2}$ ) without the use of artificial flow seeding or illumination (Schweitzer and Cowen, under review).

We present results from field measurements, made in collaboration with the United States Geological Survey (USGS) and the Department of Water Resources (DWR), in the Sacramento-San Joaquin River Delta, CA, USA, and the Finger Lakes Region of New York, USA. We compare state-of-the-art acoustic approaches used by the USGS to measurements made by our IR-QIV technique. We describe key similarities and differences relative to current visible light based techniques (e.g., Large Scale Particle Image Velocimetry, or LSPIV), methods to minimize uncertainty in the measurements, and how to use the physics of open channel flows to calculate the bathymetry from remotely measured turbulent integral length scales of the flow (Johnson and Cowen, 2016) and leverage the measured surface mean velocity field to calculate the flow rate at a river cross-section. 


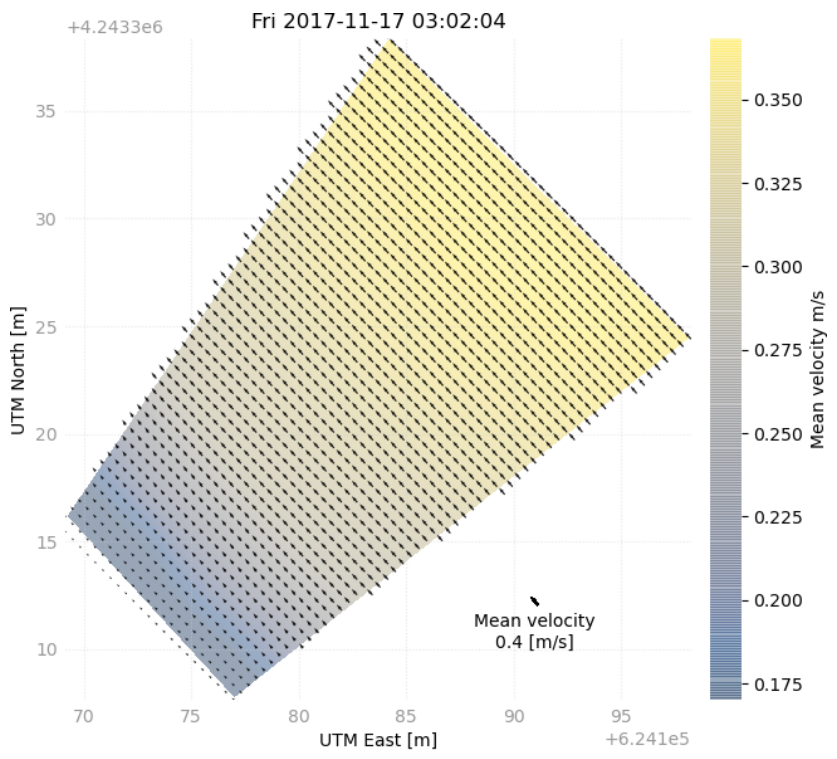

Figure 2: Five minute mean velocity field over several hundred $\mathrm{m}^{2}$ at the surface of a river, measured by IR-QIV. Background color indicates the local temporally averaged velocity magnitude. Arrows indicate the local mean velocity vector.

We describe considerations related to acquisition of infrared images for IR-QIV (camera selection, effects of environmental conditions on image quality and suitability for velocimetry), and also aspects that are relevant to large-scale, image-based, velocimetry methods, regardless of the spectral range of the images (i.e., IR or visible-light), in particular for images acquired at oblique viewing angles. These include selection of an optimal algorithm for pattern tracking (e.g., cross-correlation, minimum quadratic difference, phase-only correlation), optimal subwindow size selection based on image dynamic range and parameters such as length scales apparent in the images, georeferencing needs, and methods of quantifying uncertainty in the measurements.

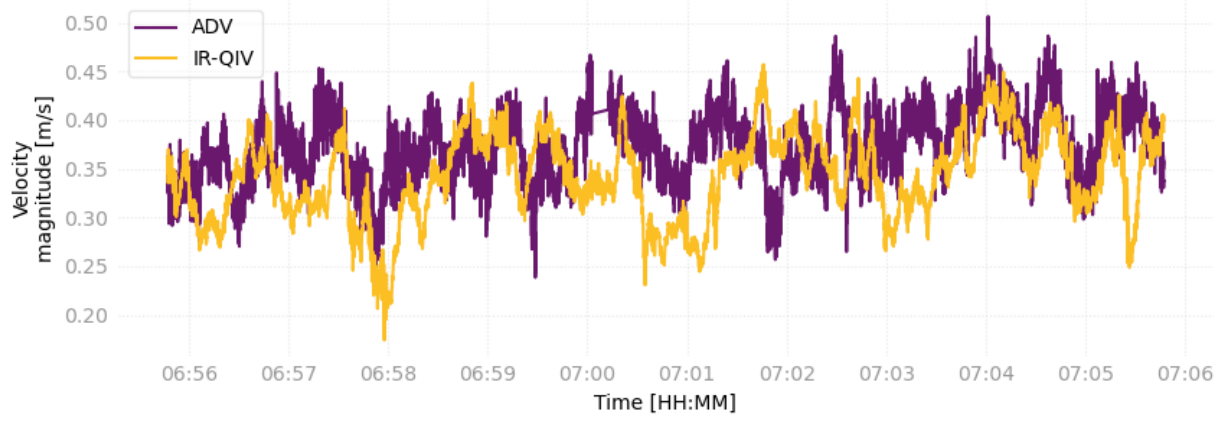

Figure 3: Comparison of ten minutes of surface velocity records made by IR-QIV and an ADV located at a depth of approximately $1 \mathrm{~m}$ and a horizontal distance of $1 \mathrm{~m}$ from the IR-QIV measurement location.

\section{Acknowledgements}

Funding for this work was provided by the California Department of Water Resources (DWR). 


\section{References}

Johnson ED and Cowen EA (2016) Remote monitoring of volumetric discharge employing bathymetry determined from surface turbulence metrics. Water Resources Research 52:2178-2193

Schweitzer SA and Cowen EA (under review) Instantaneous river-wide water surface velocity field measurements at centimeter scales using infrared quantitative image velocimetry. Water Resources Research 